\title{
Similarity Solution and Heat Transfer Characteristics for a Class of Nonlinear Convection-Diffusion Equation with Initial Value Conditions
}

\author{
Yunbin Xu \\ School of Mathematics and Statistics, Yulin University, Shaanxi, Yulin 719000, China \\ Correspondence should be addressed to Yunbin Xu; xuyunbin886@sina.com
}

Received 25 February 2019; Revised 7 May 2019; Accepted 16 May 2019; Published 23 May 2019

Academic Editor: Vincenzo Vespri

Copyright (c) 2019 Yunbin Xu. This is an open access article distributed under the Creative Commons Attribution License, which permits unrestricted use, distribution, and reproduction in any medium, provided the original work is properly cited.

\begin{abstract}
A class of nonlinear convection-diffusion equation is studied in this paper. The partial differential equation is converted into nonlinear ordinary differential equation by introducing a similarity transformation. The asymptotic analytical solutions are obtained by using double-parameter transformation perturbation expansion method (DPTPEM). The influences of convection functional coefficient $k(z)$ and power law index $n$ on the heat transport characteristics are discussed and shown graphically. The comparison with the numerical results is presented and it is found to be in excellent agreement. The method and technique used in this paper have the significance in studying other engineering problems.
\end{abstract}

\section{Introduction}

Convection-diffusion equations arose from various fields of applied sciences such as heat transfer problems in a draining film [1] or a nanofluid filled enclosure [2], radial transport in a porous medium [3], and water transport in soils [4] and have received extensive attentions during the past several decades. For convection-diffusion equations, only few cases with special initial or boundary value conditions have analytical solutions. Therefore, most of the main concerns were the study of the qualitative properties of the solutions [5-13] and numerical study [14-23] for convection-diffusion equations. However, the very important approximate solution of convection-diffusion equation has not been well solved. In this paper we present similarity solutions for a class of convection-diffusion equation, which are then solved by DPTPEM. The method was first proposed by Yan Zhang et al. [24, 25]. In this paper, we can find that the DPTPEM can be successfully used to solve the studied problem, and the approximate solutions obtained by DPTPEM agree very well the numerical solutions obtained by bvp $4 \mathrm{c}$ with Matlab [26].

\section{Mathematical Formulation}

In this paper, we consider a class of nonlinear convectiondiffusion equation as follows [20]:

$$
\begin{aligned}
& \frac{\partial u}{\partial t}=\frac{\partial}{\partial x}\left(u^{n}\left|\frac{\partial u}{\partial x}\right|^{n-1} \frac{\partial u}{\partial x}\right)+t^{-n /(n+1)} k(u) \frac{\partial u}{\partial x}, \\
&(x, t) \in G,
\end{aligned}
$$

subject to the following initial value conditions:

$$
\begin{gathered}
u(0, t)=0, \quad t>0 \\
u(x, 0)=1, \quad x>0 .
\end{gathered}
$$

where $G=\{(x, t) ; x>0, t>0\}, n$ is positive power law exponent. Convection functional coefficient $k(z)$ is assumed to be a real-valued continuous differential function defined on $[0,1], k(0)=0, k(z)>0$ for $z>0 . D(u)=-u^{n}\left|u_{x}\right|^{n-1} u_{x}$ is the heat density per unit area. 


\section{Converting into Nonlinear Ordinary Differential Equation Boundary Value Problems}

Introduce the following similarity variables [20]:

$$
\begin{aligned}
u(x, t) & =u(\eta) \\
\eta & =x t^{-1 /(n+1)} .
\end{aligned}
$$

The transformed convection-diffusion equation together with the initial value conditions given by (1)-(2) can be written as

$$
\begin{aligned}
& \left((u(\eta))^{n}\left|u^{\prime}(\eta)\right|^{n-1} u^{\prime}(\eta)\right)^{\prime} \\
& \quad+\left(\frac{\eta}{n+1}+k(u(\eta))\right) u^{\prime}(\eta)=0 \\
& u(0)=0 \\
& u(+\infty)=1 .
\end{aligned}
$$

Conversely, if $u(\eta)$ is a solution of (4)-(5), then $u(x, t)$ must be a similarity solution to (1)-(2).

Let $u=u(\eta)$ be a solution to (4)-(5). If $u(\eta)$ is strictly increasing in $[0,+\infty)$, then the function $\eta=\eta(u)$ inverse to $u=u(\eta)$ exists. Here $\eta^{\prime}(u)=\left(u^{\prime}(\eta)\right)^{-1}$ holds on $(0,1)$. Then (4) can be changed into the following nonlinear ordinary differential equation:

$$
\left(u^{n}\left(\eta^{\prime}(u)\right)^{-n}\right)^{\prime}=-\left(\frac{1}{n+1} \eta(u)+k(u)\right)
$$

and integration from $u$ to 1 yields

$$
\eta^{\prime}(u)=u\left(\int_{u}^{1}\left(\frac{1}{n+1} \eta(s)+k(s)\right) d s\right)^{-1 / n} .
$$

Set

$$
h(z)=\int_{z}^{1}\left(\frac{1}{n+1} \eta(s)+k(s)\right) d s .
$$

Combine (7) and (8) to get

$$
h^{\prime \prime}(z)=-\frac{1}{n+1} z h^{-1 / n}(z)-k^{\prime}(z)
$$

The corresponding boundary conditions are

$$
\begin{gathered}
h^{\prime}(0)=0, \\
h(1)=0 .
\end{gathered}
$$

where $h(z)$ is heat diffusion flux. The derivation process indicates that only the positive solutions of (9)-(10) are physically significant.

\section{A Brief Introduction to DPTPEM}

The method was first proposed by Yan Zhang and Liancun Zheng [24, 25].
We consider a class of ordinary differential equation initial or boundary value problem without small parameter $\varepsilon$ as follows:

$$
P: \begin{cases}L[u(x)]=f(x), & x=\left(x_{1}, x_{2}, \ldots, x_{m}\right) \in \Omega, \\ u^{(j)}(x)=a_{j}, & j=0,1, \ldots, n-1, x \in(\partial \Omega \cap \Omega) .\end{cases}
$$

where $L$ is $n$ order differential operator without small parameter $\varepsilon, a_{j}$ is a constant, and $\Omega$ is the region containing the origin.

Introduce an embedded parameter transformation as follows:

$$
u(x)=\varepsilon^{a} g(\xi)+\sum_{k=0}^{n-1} \frac{u^{(k)}(0)}{k !} x^{k}, \quad x=\varepsilon^{b} \xi
$$

where $\varepsilon$ is an artificial small parameter, $\xi$ is independent variable parameter, $g$ is dependent variable parameter, and $a$ and $b$ are undetermined constants. Substituting this transformation (12) into (11), we can obtain the following nonlinear initial value problem:

$$
P_{0}: \begin{cases}L_{\varepsilon}[g(\varepsilon, \xi)]=h(\xi), & \xi=\left(\xi_{1}, \xi_{2}, \ldots, \xi_{m}\right) \in \Omega_{1}, \\ g^{(j)}(0)=0, & j=0,1, \ldots, n-1 .\end{cases}
$$

where $h(\xi)$ is a function of $\xi, \Omega_{1}$ is the region containing the origin. The solution of $P_{0}$ is expressed in form of power series by applying perturbation expansion method

$$
g(\xi, \varepsilon)=\sum_{k=0}^{\infty} \varepsilon^{k} g_{k} \xi^{k}
$$

Combine (12) and (14) to get the solution $u$ of (11) in terms of power series. The undetermined parameters are determined according to the boundary conditions of the original problem.

The basic idea of the DPTPEM consists of three steps. Firstly, introducing an artificial small parameter $\varepsilon$, the independent variable $x$ and the unknown function $u(x)$ are transformed simultaneously, and the problem is transformed into a new one related to small parameter $\varepsilon$. Secondly, the transformed new differential equation is expanded in the form of power series of $\varepsilon$ and decomposed into the sum of several solution components. Then, by using known initial or boundary value conditions, we try to find out the solution components of each order separately and combine them to get the solution $g(\xi)$ of the new problem. Thirdly, by substituting the solution of the new equation into the transformation equation (12), the small parameter $\varepsilon$ is eliminated and the solution of the original problem is obtained.

\section{Approximate Analytical Results of (9)-(10) and Discussion}

The initial condition is assumed as follows:

$$
h(0)=\alpha
$$


In order to solve (9), we transform the dependent variable and independent variable as follows according to formula (12):

$$
\begin{aligned}
h(z) & =\varepsilon^{2} g(\xi)+\alpha, \\
z & =\varepsilon \xi,
\end{aligned}
$$

where $\varepsilon$ is an artificial small parameter.

Let $k(z)=z$ and substituting this transformation (16) into (9)-(10), we can obtain the following nonlinear initial value problems:

$$
\begin{aligned}
g^{\prime \prime}(\xi)+\frac{1}{n+1} \varepsilon \xi\left[\varepsilon^{2} g(\xi)+\alpha\right]^{-1 / n}+1 & =0 \\
g(0) & =0 \\
g^{\prime}(0) & =0 .
\end{aligned}
$$

Expanding $\left[\varepsilon^{2} g(\xi)+\alpha\right]^{-1 / n}$ in a power series development, we can get

$$
\begin{aligned}
& g^{\prime \prime}(\xi)+\frac{1}{n+1} \varepsilon \xi\left[\frac{1}{\alpha^{1 / n}}-\frac{1}{n \alpha^{(1 / n)+1}} \varepsilon^{2} g(\xi)\right. \\
& +\frac{n+1}{2 n^{2} \alpha^{1 / n+2}} \varepsilon^{4} g^{2}(\xi)-\frac{(n+1)(2 n+1)}{6 n^{3} \alpha^{1 / n+3}} \varepsilon^{6} g^{3}(\xi) \\
& \left.+\frac{(n+1)(2 n+1)(3 n+1)}{24 n^{4} \alpha^{1 / n+4}} \varepsilon^{8} g^{4}(\xi)+\cdots\right]+1=0
\end{aligned}
$$

The solution of (19) can be obtained by expanding $g(\xi)$ in a power series development near $\varepsilon=0$ as follows:

$$
\begin{aligned}
g(\xi)= & g_{0}(\xi)+\varepsilon g_{1}(\xi)+\varepsilon^{2} g_{2}(\xi)+\varepsilon^{3} g_{3}(\xi) \\
& +\varepsilon^{4} g_{4}(\xi)+\varepsilon^{5} g_{5}(\xi)+\varepsilon^{6} g_{6}(\xi)+\varepsilon^{7} g_{7}(\xi) \\
& +\varepsilon^{8} g_{8}(\xi)+\varepsilon^{9} g_{9}(\xi)+\varepsilon^{10} g_{10}(\xi)+\cdots
\end{aligned}
$$

Substituting (20) into (19) and equating the coefficients of $\varepsilon^{i}$, we can get the following expressions:

$$
\begin{aligned}
& \varepsilon^{0}: g_{0}^{\prime \prime}(\xi)+1=0, \\
& \varepsilon^{1}: g_{1}^{\prime \prime}(\xi)+\frac{\xi}{(n+1) \alpha^{1 / n}}=0, \\
& \varepsilon^{2}: g_{2}^{\prime \prime}(\xi)=0, \\
& \varepsilon^{3}: g_{3}^{\prime \prime}(\xi)-\frac{\xi g_{0}(\xi)}{n(n+1) \alpha^{1 / n+1}}=0, \\
& \varepsilon^{4}: g_{4}^{\prime \prime}(\xi)-\frac{\xi g_{1}(\xi)}{n(n+1) \alpha^{1 / n+1}}=0, \\
& \varepsilon^{5}: g_{5}^{\prime \prime}(\xi)-\frac{\xi g_{2}(\xi)}{n(n+1) \alpha^{1 / n+1}}+\frac{\xi g_{0}^{2}(\xi)}{2 n^{2} \alpha^{1 / n+2}}=0, \\
& \varepsilon^{6}: g_{6}^{\prime \prime}(\xi)-\frac{\xi g_{3}(\xi)}{n(n+1) \alpha^{1 / n+1}}+\frac{\xi g_{0}(\xi) g_{1}(\xi)}{n^{2} \alpha^{1 / n+2}}=0,
\end{aligned}
$$

$$
\begin{aligned}
& \varepsilon^{7}: g_{7}^{\prime \prime}(\xi)-\frac{\xi g_{4}(\xi)}{n(n+1) \alpha^{1 / n+1}}+\frac{\xi\left[2 g_{0}(\xi) g_{2}(\xi)+g_{1}^{2}(\xi)\right]}{2 n^{2} \alpha^{1 / n+2}} \\
& -\frac{(2 n+1) \xi g_{0}^{3}(\xi)}{6 n^{3} \alpha^{1 / n+3}}=0, \\
& \varepsilon^{8}: g_{8}^{\prime \prime}(\xi)-\frac{\xi g_{5}(\xi)}{n(n+1) \alpha^{1 / n+1}} \\
& +\frac{\xi\left[g_{0}(\xi) g_{3}(\xi)+g_{1}(\xi) g_{2}(\xi)\right]}{n^{2} \alpha^{1 / n+2}} \\
& -\frac{(2 n+1) \xi g_{0}^{2}(\xi) g_{1}(\xi)}{2 n^{3} \alpha^{1 / n+3}}=0, \\
& \varepsilon^{9}: g_{9}^{\prime \prime}(\xi)-\frac{\xi g_{6}(\xi)}{n(n+1) \alpha^{1 / n+1}} \\
& +\frac{\xi\left[2 g_{0}(\xi) g_{4}(\xi)+2 g_{1}(\xi) g_{3}(\xi)+g_{2}^{2}(\xi)\right]}{2 n^{2} \alpha^{1 / n+2}} \\
& -\frac{(2 n+1) \xi\left[g_{0}^{2}(\xi) g_{2}(\xi)+g_{0}(\xi) g_{1}^{2}(\xi)\right]}{2 n^{3} \alpha^{1 / n+3}} \\
& +\frac{(2 n+1)(3 n+1) \xi g_{0}^{4}(\xi)}{24 n^{4} \alpha^{1 / n+4}}=0, \\
& \varepsilon^{10}: g_{10}^{\prime \prime}(\xi)-\frac{\xi g_{7}(\xi)}{n(n+1) \alpha^{1 / n+1}} \\
& +\frac{\xi\left[g_{0}(\xi) g_{5}(\xi)+g_{1}(\xi) g_{4}(\xi)+g_{2}(\xi) g_{3}(\xi)\right]}{n^{2} \alpha^{1 / n+2}} \\
& -\frac{(2 n+1) \xi\left[3 g_{0}^{2}(\xi) g_{3}(\xi)+6 g_{0}(\xi) g_{1}(\xi) g_{2}(\xi)+g_{1}^{3}(\xi)\right]}{6 n^{3} \alpha^{1 / n+3}} \\
& +\frac{(2 n+1)(3 n+1) \xi g_{0}^{3}(\xi) g_{1}(\xi)}{6 n^{4} \alpha^{1 / n+4}}=0,
\end{aligned}
$$

Then, we can obtain $g_{i}(\xi)(i=0,1,2, \ldots)$ in the following.

$$
\begin{aligned}
& g_{0}(\xi)=-\frac{1}{2} \xi^{2}, \\
& g_{1}(\xi)=-\frac{1}{6(n+1) \alpha^{1 / n}} \xi^{3}, \\
& g_{2}(\xi)=0 \\
& g_{3}(\xi)=-\frac{1}{40 n(n+1) \alpha^{1 / n+1}} \xi^{5}, \\
& g_{4}(\xi)=-\frac{1}{180 n(n+1)^{2} \alpha^{2 / n+1}} \xi^{6}, \\
& g_{5}(\xi)=-\frac{1}{336 n^{2} \alpha^{1 / n+2}} \xi^{7}, \\
& g_{6}(\xi)=-\frac{10 n+13}{6720 n^{2}(n+1)^{2} \alpha^{2 / n+2}} \xi^{8}, \\
& g_{7}(\xi)=-\left[\frac{5 n+7}{25920 n^{2}(n+1)^{3} \alpha^{3 / n+2}}\right.
\end{aligned}
$$




$$
\begin{gathered}
\left.+\frac{2 n+1}{3456 n^{3} \alpha^{1 / n+3}}\right] \xi^{9}, \\
g_{8}(\xi)=-\frac{70 n+61}{151200(n+1) n^{3} \alpha^{2 / n+3}} \xi^{10}, \\
g_{9}(\xi)=-\left[\frac{280 n^{2}+590 n+319}{2217600 n^{3}(n+1)^{3} \alpha^{3 / n+3}}\right. \\
\left.+\frac{6 n^{2}+5 n+1}{42240 n^{4} \alpha^{1 / n+4}}\right] \xi^{11}, \\
g_{10}(\xi)=-\left[\frac{15120 n^{2}+18636 n+6078}{95800320(n+1) n^{4} \alpha^{2 / n+4}}\right. \\
\left.+\frac{1200 n^{2}+2670 n+1530}{102643200 n^{3}(n+1)^{4} \alpha^{4 / n+3}}\right] \xi^{12}, \\
\ldots . .
\end{gathered}
$$

Combine (16), (20), and the above $g_{i}(\xi)(i=0,1,2, \ldots)$ to get solution $h(z)$ of (9)-(10).

$$
\begin{aligned}
h & =\alpha-\frac{1}{2} z^{2}-\frac{1}{6(n+1) \alpha^{1 / n}} z^{3}-\frac{1}{40 n(n+1) \alpha^{1 / n+1}} \\
& \cdot z^{5}-\frac{1}{180 n(n+1)^{2} \alpha^{2 / n+1}} z^{6}-\frac{1}{336 n^{2} \alpha^{1 / n+2}} z^{7} \\
& -\frac{10 n+13}{6720 n^{2}(n+1)^{2} \alpha^{2 / n+2}} z^{8} \\
& -\left[\frac{5 n+7}{25920 n^{2}(n+1)^{3} \alpha^{3 / n+2}}+\frac{2 n+1}{3456 n^{3} \alpha^{1 / n+3}}\right] z^{9} \\
& -\frac{70 n+61}{151200(n+1) n^{3} \alpha^{2 / n+3}} z^{10} \\
& -\left[\frac{280 n^{2}+590 n+319}{2217600 n^{3}(n+1)^{3} \alpha^{3 / n+3}}+\frac{6 n^{2}+5 n+1}{42240 n^{4} \alpha^{1 / n+4}}\right] \\
& \cdot z^{11}-\left[\frac{15120 n^{2}+18636 n+6078}{95800320(n+1) n^{4} \alpha^{2 / n+4}}\right. \\
& \left.+\frac{1200 n^{2}+2670 n+1530}{102643200 n^{3}(n+1)^{4} \alpha^{4 / n+3}}\right] z^{12}+\cdots
\end{aligned}
$$

Similarly, let $k(z)=z^{2}$; we obtain approximate analytical solution of (9)-(10) as follows:

$$
\begin{aligned}
h & =\alpha-\left[\frac{1}{3}+\frac{1}{6(n+1) \alpha^{1 / n}}\right] z^{3} \\
& -\left[\frac{1}{90 n(n+1) \alpha^{1 / n+1}}+\frac{1}{180 n(n+1)^{2} \alpha^{2 / n+1}}\right] z^{6}
\end{aligned}
$$

TABLE 1: Values of $\alpha$ for $n=0.2,0.6,1.0,2.5,5.0,10.0$, and $k(z)=z$.

\begin{tabular}{lcc}
\hline$n$ & $k(z)$ & $\alpha$ \\
\hline 0.2 & $z$ & 1.013 \\
0.6 & $z$ & 0.7815 \\
1.0 & $z$ & 0.6792 \\
2.5 & $z$ & 0.5701 \\
5.0 & $z$ & 0.5342 \\
10.0 & $z$ & 0.5169 \\
\hline
\end{tabular}

$$
\begin{aligned}
& -\left[\frac{1}{1296 n^{2} \alpha^{1 / n+2}}+\frac{5 n+6}{6480 n^{2}(n+1)^{2} \alpha^{2 / n+2}}\right. \\
& \left.+\frac{5 n+7}{25920 n^{2}(n+1)^{3} \alpha^{3 / n+2}}\right] z^{9}-\left[\frac{2 n+1}{21384 n^{3} \alpha^{1 / n+3}}\right. \\
& +\frac{120 n+89}{855360(n+1) n^{3} \alpha^{2 / n+3}} \\
& +\frac{60 n^{2}+119 n+60}{855360 n^{3}(n+1)^{3} \alpha^{3 / n+3}} \\
& \left.+\frac{40 n^{2}+89 n+51}{3421440 n^{3}(n+1)^{4} \alpha^{4 / n+3}}\right] z^{12}+\cdots
\end{aligned}
$$

Let $k(z)=z^{3}$; approximate analytical solution of (9)-(10) is presented as follows:

$$
\begin{aligned}
h= & \alpha-\frac{1}{6(n+1) \alpha^{1 / n}} z^{3}-\frac{1}{4} z^{4} \\
& -\frac{1}{180 n(n+1)^{2} \alpha^{2 / n+1}} z^{6} \\
& -\frac{1}{168 n(n+1) \alpha^{1 / n+1}} z^{7} \\
& -\frac{5 n+7}{25920 n^{2}(n+1)^{3} \alpha^{3 / n+2}} z^{9} \\
& -\frac{7 n+8}{15120 n^{2}(n+1)^{2} \alpha^{2 / n+2}} z^{10}-\frac{1}{3520 n^{2} \alpha^{1 / n+2}} z^{11} \\
& -\frac{40 n^{2}+89 n+51}{3421440 n^{3}(n+1)^{4} \alpha^{4 / n+3}} z^{12}+\cdots
\end{aligned}
$$

It is obvious that we can promptly obtain the value of $\alpha$ by applying $h(1)=0$ in (23)-(25) for each fixed $n$. Based on (23)(25) and the corresponding $\alpha$ we can easily obtain the graph of the heat diffusion flux distribution for different $n$ and $k(z)$. The results are presented in Tables 1-5 and Figures 1-5.

It can be seen from Figures 1-3 that the heat diffusion flux $h(z)$ decreases with increase of $n$ for specific $k(z)$, the physical meaning is that heat diffusion flux $h(z)$ is a decreasing function of $n$, which means that the profiles exhibited by a 




FIGURE 1: Flux distribution for $n=0.2,0.6,1.0,2.55 .0,10.0, k(z)=z$.

TABLE 2: Values of $\alpha$ for $n=0.2,0.6,1.0,2.5,5.0,10.0$, and $k(z)=z^{2}$.

\begin{tabular}{lcc}
\hline$n$ & $k(z)$ & $\alpha$ \\
\hline 0.2 & $z^{2}$ & 0.9122 \\
0.6 & $z^{2}$ & 0.6532 \\
1.0 & $z^{2}$ & 0.5352 \\
2.5 & $z^{2}$ & 0.4090 \\
5.0 & $z^{2}$ & 0.3691 \\
10.0 & $z^{2}$ & 0.3506 \\
\hline
\end{tabular}

TABLE 3: Values of $\alpha$ for $n=0.2,0.6,1.0,2.5,5.0,10.0$, and $k(z)=z^{3}$.

\begin{tabular}{lcc}
\hline$n$ & $k(z)$ & $\alpha$ \\
\hline 0.2 & $z^{3}$ & 0.8643 \\
0.6 & $z^{3}$ & 0.5927 \\
1.0 & $z^{3}$ & 0.4670 \\
2.5 & $z^{3}$ & 0.3300 \\
5.0 & $z^{3}$ & 0.2870 \\
10.0 & $z^{3}$ & 0.2676 \\
\hline
\end{tabular}

TABLE 4: Values of $\alpha$ for $n=2.0$ and $k(z)=z, z^{2}, z^{3}$.

\begin{tabular}{lcc}
\hline$n$ & $k(z)$ & $\alpha$ \\
\hline 2.0 & $z$ & 0.5885 \\
2.0 & $z^{2}$ & 0.4300 \\
2.0 & $z^{3}$ & 0.3529 \\
\hline
\end{tabular}

big power law index $n$ possess a smaller diffusion. Figures 4 and 5 indicate the heat diffusion flux $h(z)$ decrease with the decrease of $k(z)$.

In order to verify the efficiency and reliability of approximate analytical solution obtained by using DPTPEM, a
TABLE 5: Values of $\alpha$ for $n=8.0$ and $k(z)=z, z^{2}, z^{3}$.

\begin{tabular}{lcc}
\hline$n$ & $k(z)$ & $\alpha$ \\
\hline 8.0 & $z$ & 0.5212 \\
8.0 & $z^{2}$ & 0.3551 \\
8.0 & $z^{3}$ & 0.2723 \\
\hline
\end{tabular}

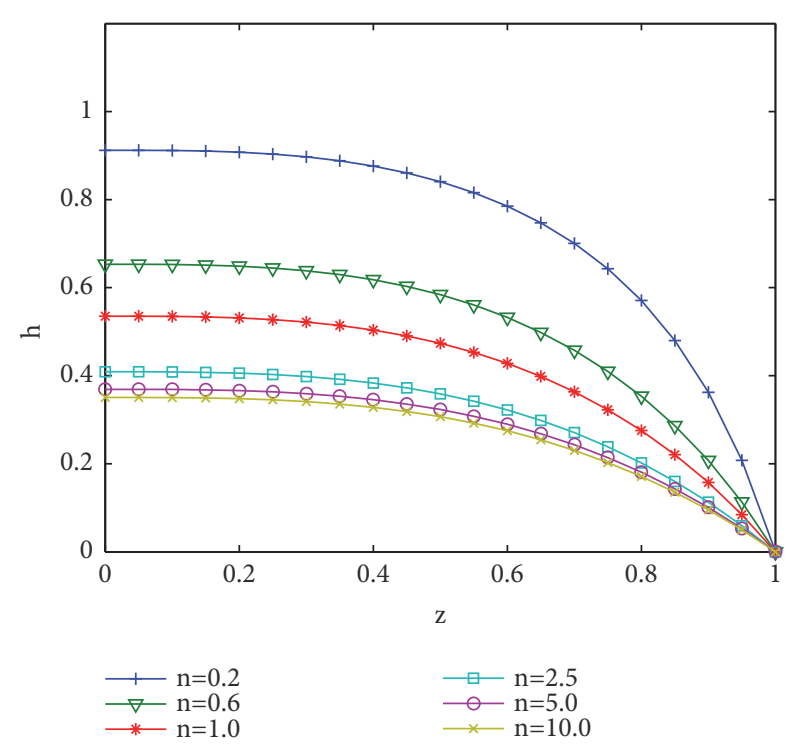

FIGURE 2: Flux distribution for $n=0.2,0.6,1.0,2.55 .0,10.0, k(z)=$ $z^{2}$.

comparison of approximate analytical solution and numerical one obtained by bvp4c with Matlab is presented in Tables 6 and 7 and Figures 6 and 7. It is obvious that excellent 


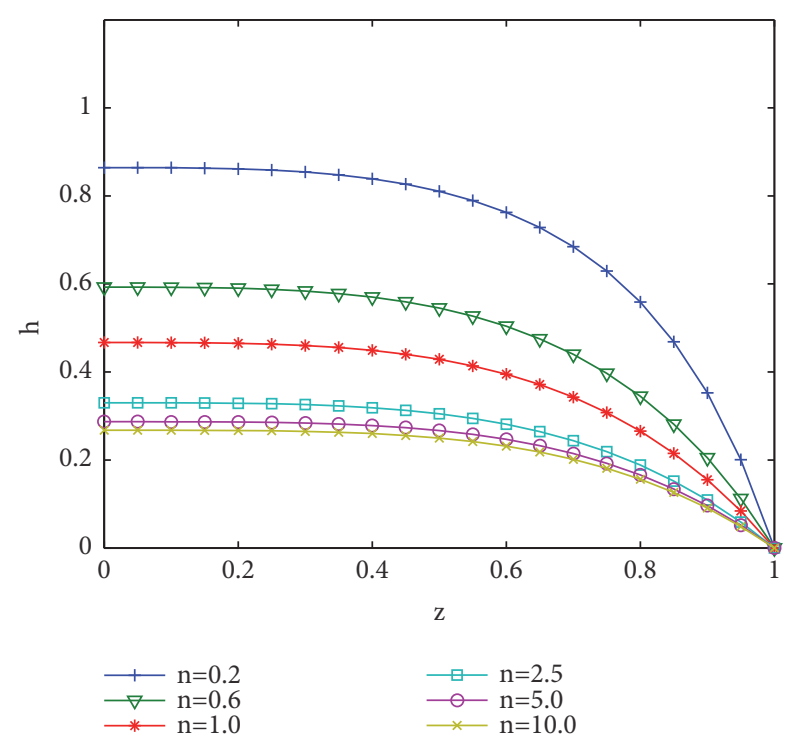

FIGURE 3: Flux distribution for $n=0.2,0.6,1.0,2.55 .0,10.0, k(z)=$ $z^{3}$.

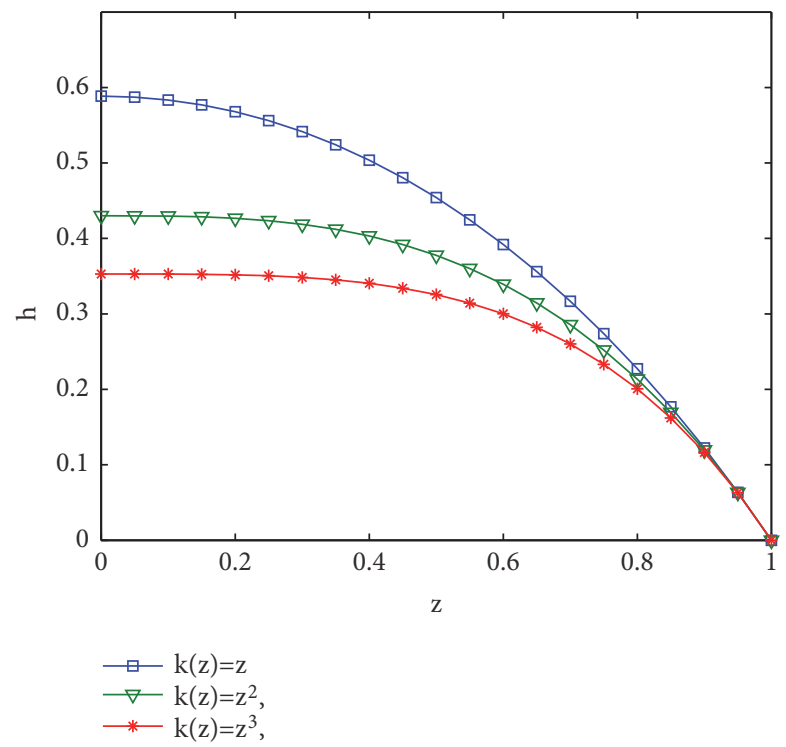

FIGURE 4: Flux distribution for $n=2.0, k(z)=z, z^{2}, z^{3}$.

agreement exists for approximate analytical solution and numerical one.

\section{Conclusions}

In the paper, a class of nonlinear convection-diffusion was studied. The partial differential equation and corresponding initial value conditions were transformed into a class of singular nonlinear boundary value problems of ordinary differential equation when similarity variables were introduced. An efficient approximate analytical method named DPTPEM was applied to solve these nonlinear problems. The reliability and effectiveness of the DPTPEM were verified by comparing approximate results with the numerical solutions. The effects

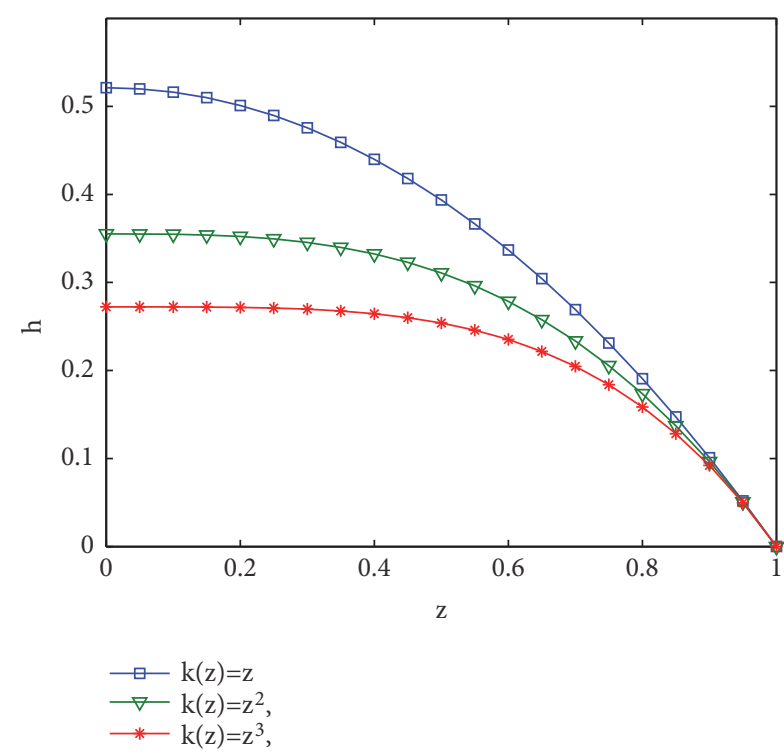

Figure 5: Flux distribution for $n=8.0, k(z)=z, z^{2}, z^{3}$.

TABLE 6: Comparison of $h(z)$ for $n=10$ and $k(z)=z$.

\begin{tabular}{lcc}
\hline$z$ & DPTPEM & bvp4c \\
\hline 0.0 & 0.5169 & 0.5169 \\
0.1 & 0.5119 & 0.5119 \\
0.2 & 0.4968 & 0.4968 \\
0.3 & 0.4715 & 0.4715 \\
0.4 & 0.4359 & 0.4359 \\
0.5 & 0.3899 & 0.3899 \\
0.6 & 0.3334 & 0.3334 \\
0.7 & 0.2663 & 0.2663 \\
0.8 & 0.1884 & 0.1884 \\
0.9 & 0.0997 & 0.0997 \\
1.0 & 0 & 0 \\
\hline
\end{tabular}

TABLE 7: Comparison of $h(z)$ for $n=2$ and $k(z)=z^{3}$.

\begin{tabular}{lcc}
\hline$z$ & DPTPEM & $b v p 4 c$ \\
\hline 0.0 & 0.3529 & 0.3551 \\
0.1 & 0.3528 & 0.3550 \\
0.2 & 0.3518 & 0.3540 \\
0.3 & 0.3483 & 0.3506 \\
0.4 & 0.3405 & 0.3427 \\
0.5 & 0.3255 & 0.3278 \\
0.6 & 0.3000 & 0.3023 \\
0.7 & 0.2601 & 0.2624 \\
0.8 & 0.2008 & 0.2031 \\
0.9 & 0.1164 & 0.1186 \\
1.0 & 0 & 0 \\
\hline
\end{tabular}

of convection functional coefficient $k(z)$ and power law index $n$ on transfer behavior were presented. The results show that heat diffusion flux $h(z)$ is an increasing function 


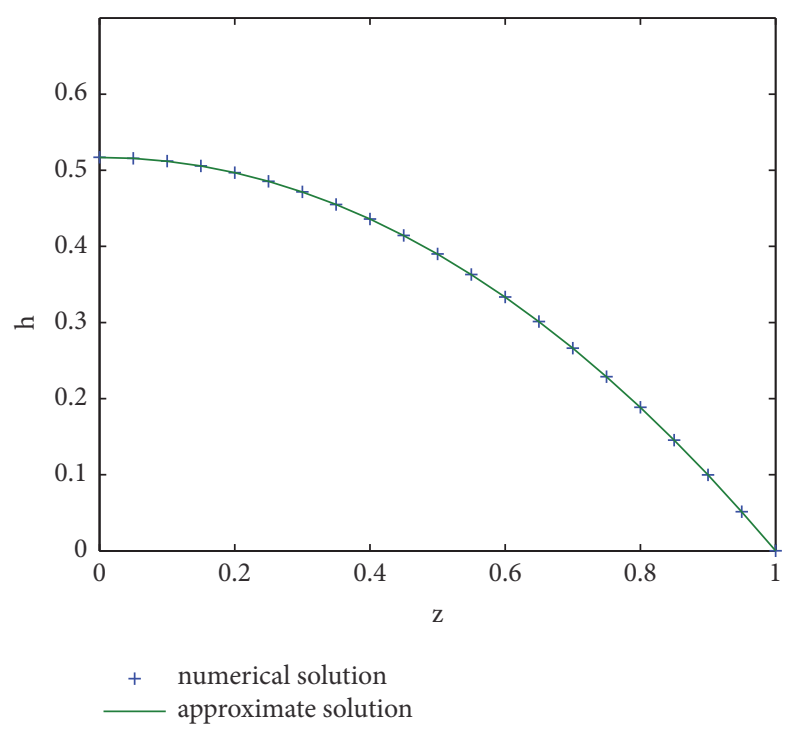

FIGURE 6: The comparison of the results of approximate analytical solution and numerical solution for $n=10.0, k(z)=z$.

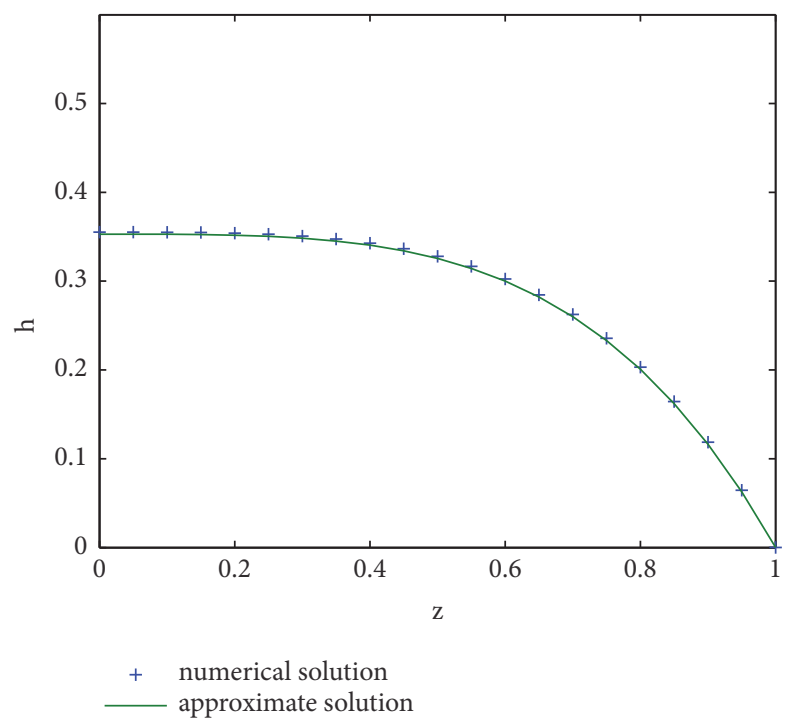

FIgURE 7: The comparison of the results of approximate analytical solution and numerical solution for $n=2, k(z)=z^{3}$.

of convection functional coefficient $k(z)$ and a decreasing function of power law index $n$.

\section{Data Availability}

The data used to support the findings of this study are available from the corresponding author upon request.

\section{Conflicts of Interest}

The author declares that there are no conflicts of interest.

\section{Acknowledgments}

The research was supported by a grant from the National Natural Science Foundation of China (no. 11501496) and the Natural Science Basic Research Plan in Shaanxi Province of China (no. 2014JQ2-1003).

\section{References}

[1] J. Isenberg and C. Gutfinger, "Heat transfer to a draining film," International Journal of Heat and Mass Transfer, vol. 16, no. 2, pp. 505-512, 1973.

[2] M. Sheikholeslami, R. Ellahi, M. Hassan, and S. Soleimani, "A study of natural convection heat transfer in a nanofluid filled enclosure with elliptic inner cylinder," International Journal of Numerical Methods for Heat \& Fluid Flow, vol. 24, no. 8, pp. 1906-1927, 2014.

[3] E. J. Veling, "Radial transport in a porous medium with dirichlet, neumann and robin-type inhomogeneous boundary values and general initial data: analytical solution and evaluation," Journal of Engineering Mathematics, vol. 75, pp. 173-189, 2012.

[4] J.-Y. Parlange, "Water transport in soils.," Annual review of fluid mechanics, Vol. 12, pp. 77-102, 1980.

[5] X. Yu and Q. S. Zhang, "Asymptotically self-similar solution for the convection-diffusion equation," Applied Mechanics and Materials, vol. 623, pp. 97-100, 2014.

[6] M. o. Krupski, "Convection-diffusion equations with random initial conditions," Journal of Mathematical Analysis and Applications, vol. 470, no. 2, pp. 1194-1221, 2019.

[7] B. I. Olajuwon and A. O. Popoola, "On self-similar solution of non-linear diffusion equation with convection term," Journal of Mathematics and Statistics, vol. 2, no. 4, pp. 453-456, 2006.

[8] G. Lu and L. Jiang, "A remark on removable singularity for nonlinear convection-diffusion equation," Nonlinear Analysis: Real World Applications, vol. 27, pp. 1-25, 2016.

[9] L. I. Ignat and D. Stan, "Asymptotic behavior of solutions to fractional diffusion-convection equations," Journal of The London Mathematical Society-Second Series, vol. 97, no. 2, pp. 258-281, 2018.

[10] A. Gmira and L. Veron, "Large time behaviour of the solutions of a semilinear parabolic equation in $\mathbb{R}^{N}$," Journal of Differential Equations, vol. 53, no. 2, pp. 258-276, 1984.

[11] M. Escobedo and E. Zuazua, "Large time behavior for convection-diffusion equations in $\mathrm{R}^{N}$," Journal of Functional Analysis, vol. 100, pp. 119-161, 1991.

[12] K. Kalli and K. N. Soltanov, "Existence and behavior of solutions for convection-diffusion equations with third type boundary condition," TWMS Journal of Pure and Applied Mathematics, vol. 8, no. 2, pp. 209-222, 2017.

[13] L. Greco, G. Moscariello, and G. Zecca, "Very weak solutions to elliptic equations with singular convection term," Journal of Mathematical Analysis and Applications, vol. 457, no. 2, pp. 13761387, 2018.

[14] S. V. Siryk, "A note on the application of the GuermondPasquetti mass lumping correction technique for convectiondiffusion problems," Journal of Computational Physics, vol. 376, pp. 1273-1291, 2019.

[15] R. Bürger and I. Kröker, "Computational uncertainty quantification for some strongly degenerate parabolic convectiondiffusion equations," Journal of Computational and Applied Mathematics, vol. 348, pp. 490-508, 2019. 
[16] R. Costa, J. M. Nóbrega, S. Clain, G. J. Machado, and R. Loubère, "Very high-order accurate finite volume scheme for the convection-diffusion equation with general boundary conditions on arbitrary curved boundaries," International Journal for Numerical Methods in Engineering, vol. 117, no. 2, pp. 188220, 2019.

[17] Z. Chen, S.-H. Chou, and D. Y. Kwak, "Characteristic-mixed covolume methods for advection-dominated diffusion problems," Numerical Linear Algebra with Applications, vol. 13, no. 9, pp. 677-697, 2006.

[18] S. May, "Spacetime discontinuous Galerkin methods for convection-diffusion equations," Bulletin of the Brazilian Mathematical Society, vol. 47, no. 2, pp. 561-573, 2016.

[19] A. V. Gladky, "Stability of difference splitting schemes for convection diffusion equation," Cybernetics and Systems Analysis, vol. 53, no. 2, pp. 193-203, 2017.

[20] L. C. Zheng, X. X. Zhang, and J. C. He, "Free boundary value problems for a class of generalized diffusion equation," Journal of University of Science and Technology Beijing, vol. 9, no. 6, pp. 422-425, 2002.

[21] M. R. Sidi Ammi and I. Jamiai, "Finite difference and Legendre spectral method for a time-fractional diffusion-convection equation for image restoration," Discrete and Continuous Dynamical Systems - Series S, vol. 11, no. 1, pp. 103-117, 2018.

[22] R. C. Lin, X. Ye, S. Zhang, and P. Zhu, "A weak Galerkin finite element method for singularly perturbed convection-diffusionreaction problems," SIAM Journal on Numerical Analysis, vol. 56, no. 3, pp. 1482-1497, 2018.

[23] M. Saqib, S. Hasnain, and D. S. Mashat, "Computational solutions of two dimensional convection diffusion equation using crank-nicolson and time efficient ADI," American Journal of Computational Mathematics, vol. 7, no. 3, pp. 208-227, 2017.

[24] Y. Zhang, L. C. Zheng, and X. X. Zhang, "Analytical approximate solution for Marangoni convection in a liquid layer with coupled boundary conditions," Acta Physica Sinica, vol. 58, no. 8, pp. 5501-5506, 2009.

[25] L. C. Zheng and X. X. Zhang, Modern Analysis Methods in Nonlinear Partial Differential Equations, Science Press, Beijing, China, 2011.

[26] X. Si, H. Li, Y. Shen, and L. Zheng, "Effects of nonlinear velocity slip and temperature jump on pseudo-plastic power-law fluid over moving permeable surface in presence of magnetic field," Applied Mathematics and Mechanics-English Edition, vol. 38, no. 3, pp. 333-342, 2017. 


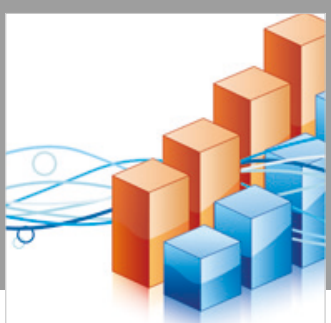

Advances in

Operations Research

\section{-n-m}

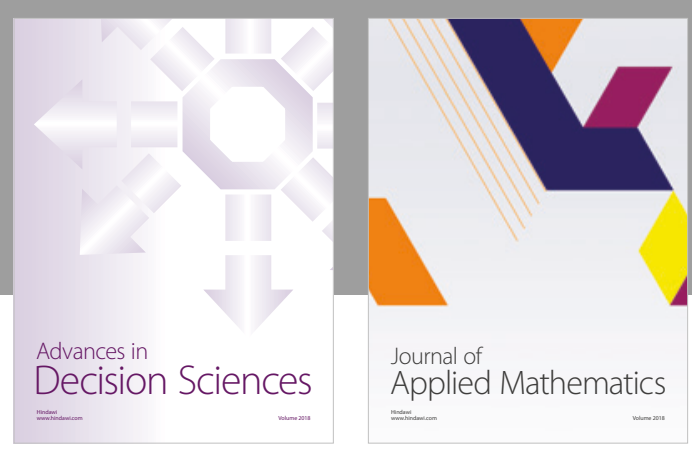

Journal of

Applied Mathematics
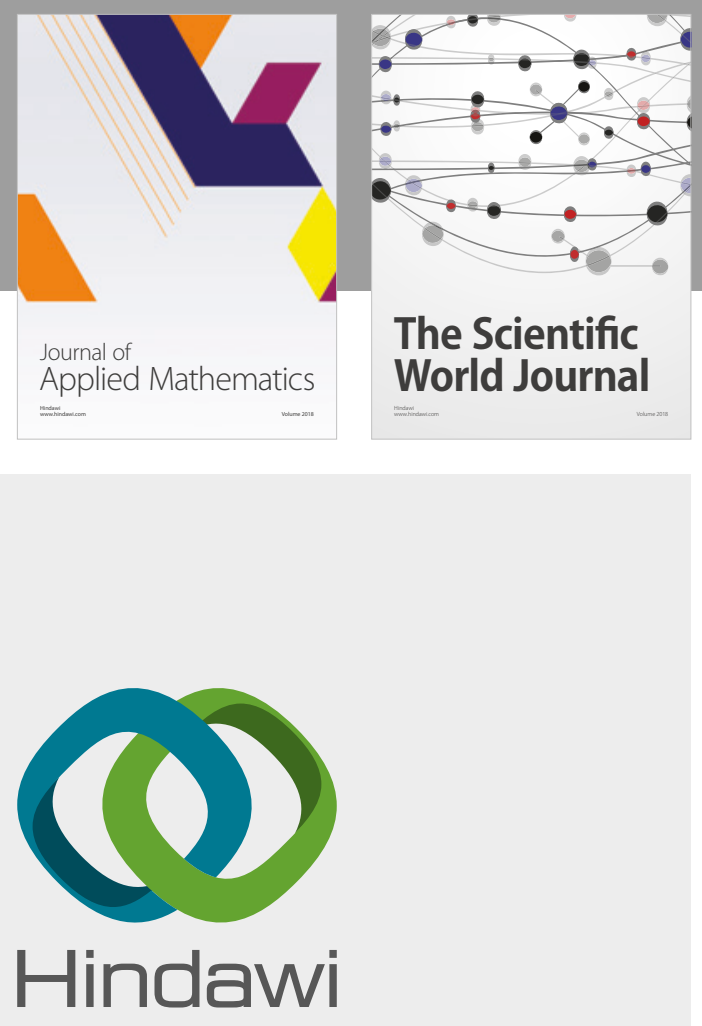

Submit your manuscripts at

www.hindawi.com

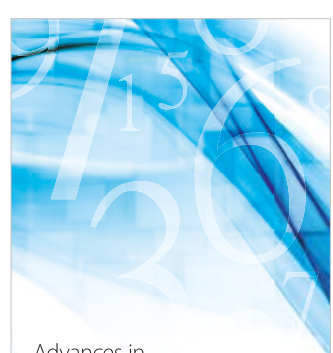

Advances in
Numerical Analysis
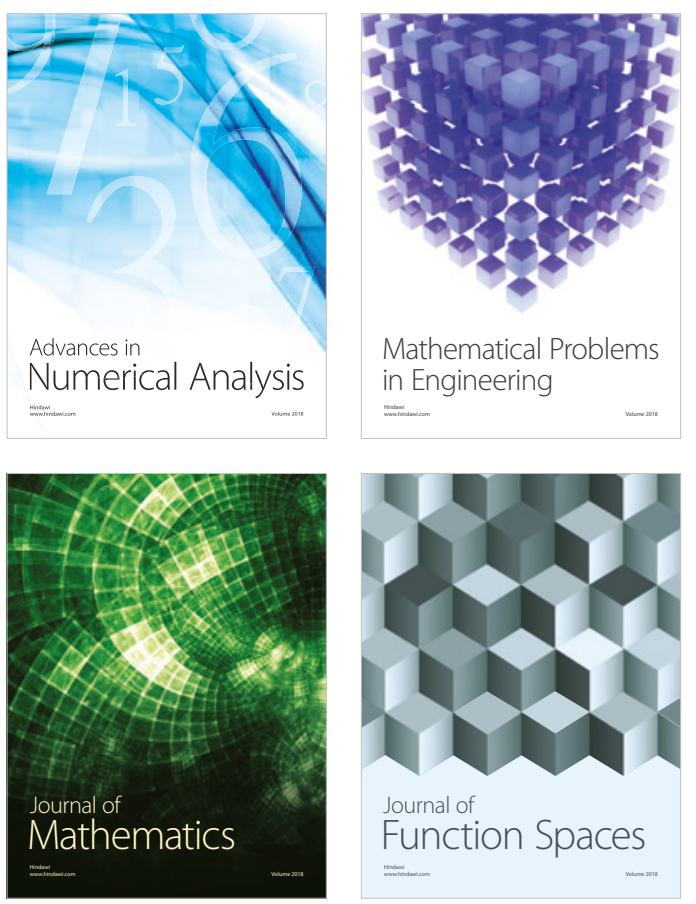

Mathematical Problems in Engineering

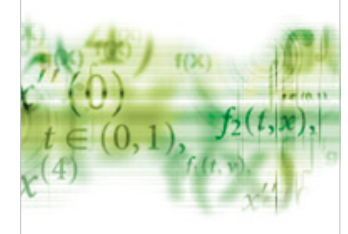

International Journal of

Differential Equations

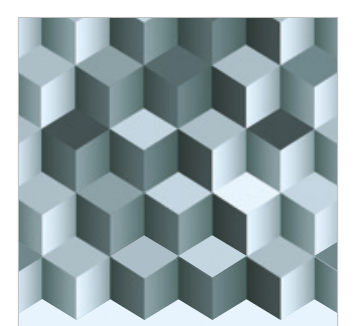

Journal of

Function Spaces

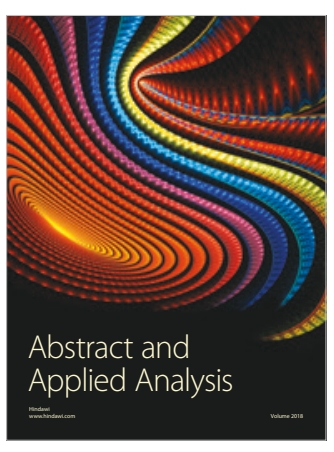

The Scientific

World Journal

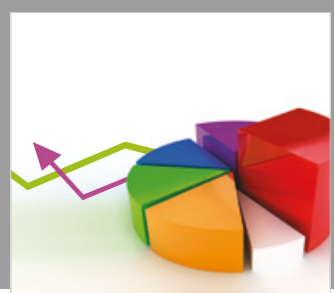

Journal of

Probability and Statistics
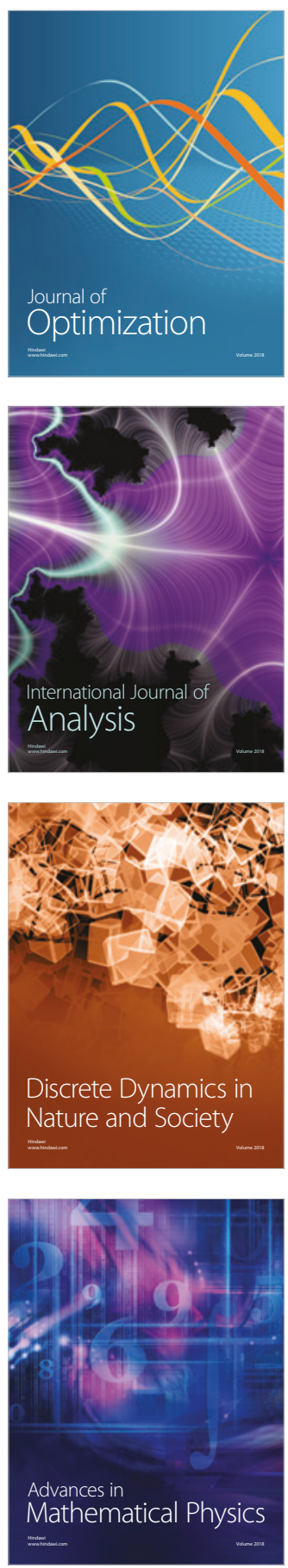